\title{
Aplicación de los factores de participación y del método de MW-Milla en la asignación de cargos por uso de redes de transmisión en mercados de electricidad
}

\author{
L. Alba-Gómez, J.H. Tovar-Hernández y G. Gutiérrez-Alcaraz \\ Programa de Graduados e Investigación en Ingeniería Eléctrica \\ Departamento de Ingeniería Eléctrica y Electrónica \\ Instituto Tecnológico de Morelia, Morelia Michoacán, México \\ E-mails:horaciotovar@mexico.com,ggutier@itmorelia.edu.mx
}

(Recibido: mayo de 2006; aceptado: septiembre de 2006)

\begin{abstract}
Resumen
Este trabajo presenta la asignación de costos por uso de red mediante la aplicación de Factores de Participación (FP) y el método de MW-Milla. Los FP clásicos son calculados a partir del modelo lineal de flujos de potencia para lo que se requiere de establecer un nodo de referencia a fin de eliminar la singularidad de la matriz de coeficientes. Por lo tanto, los FP son dependientes de la asignación del nodo de referencia. Dos métodos alternativos para evitar la dependencia del nodo compensador en la obtención de los factores de participación son presentados.
\end{abstract}

Descriptores: Factores de participación, mercados de electricidad.

\begin{abstract}
Use of net work al lo ca tion costs by shift fac tors and MW-Mile method is re ported in this paper. Con ven tional shift fac tors are com puted based on DC power flow. DC power flow requires to se lect ing a slack bus in or der to avoid ma trix sin gu lar ity. There fore, shift factors are slack bus de pend ent. In or der to evade slack bus de pend ency, two ap proaches are considered.
\end{abstract}

Keywords: Falta traducción, Falta traducción,Falta traducción,Falta traducción.

\section{Introducción}

La organización general previa a la reestructuración fue basada en compañías verticalmente integradas, responsables de las actividades de generación, transmisión, distribución y comercialización de la energía eléctrica.

Con la reestructuración, las diferentes actividades se han desagregado, originando una estructura horizontal, en donde varias empresas pueden ofertar el mismo servicio dentro de cada actividad. Sin embargo, las actividades de transmisió n y distribución continú an operando bajo un esquema de monopolio regional.

Desde un punto de vista de asignación de cargos fijos por el servicio de transmisión, las metodologías que se desarrollaron hace algunos años se han basado en la existencia de transacciones bilaterales. Si la estructura del mercado es tal que no permite establecer una relación directa entre compradores y vendedores a travé s de una cantidad específica de MW, entonces, los conceptos anteriores son evaluados estableciendo responsabilidades por 
separado a generadores y consumidores. Bajo este entorno, normalmente la única componente de costos, desglosada en varios rubros, es la relacionada con el uso de la red de transmisión. Aquí, el problema consiste en definir los costos por uso de red y desarrollar un procedimiento equitativo de asignación entre todos los agentes participantes en el mercado. Como parte de este proceso, primeramente se deben definir los costos relacionados con la infraestructura de transmisión. Posteriormente, se definen los agentes del mercado que deberán pagar los cargos correspondientes al servicio de transporte; tales agentes pueden ser los generadores o los consumidores, o ambos. En el caso de que deban pagar únicamente, ya sean generadores o consumidores, entonces, la metodología basada en flujos puede aplicar métodos de descomposición proporcional (Bialek, 1997) y (Strbac et al., 1997) o factores de participación generalizados (Rudnick et al., 1995). Lo más común es que ambos grupos de agentes paguen por el servicio de transmisión y estos métodos se pueden aplicar, definiendo previamente el porcentaje en que cada grupo habrá de cubrir los cargos totales del servicio de transmisión, lo cual puede conducir a la aplicación de criterios que pueden ser arbitrarios (Alexander y Muñ oz, 2006). Cuando es difícil definir tal porcentaje, una solución adecuada es la utilización del modelo de FP convencionales, los cuales permiten definir de manera automática los cargos por uso de la red de transmisión a todos aquellos participantes del mercado que utilizan la red de transmisión, ya sea generando o absorbiendo energía a través de ella. Sin embargo, este modelo también presenta inconvenientes. El modelo convencional para el cálculo de los FP se basa en el modelo lineal de flujos de potencia, conocido como flujos de C.D. El cálculo de FP requiere establecer un nodo de referencia, a fin de eliminar la singularidad de la matriz de coeficientes asociados al modelo. Por lo tan to, los FP son dependientes de la asignación del nodo de referencia. Resulta que si $j$ es el nodo de referencia, entonces todos los FP con respecto a $j$ son cero. Esto significa que, para la asignación de cargos por uso de redes de transmisión, el nodo de referencia no tendría cargo alguno por el uso de la red de transmisión. Existen dos métodos para reducireste inconveniente :

a) intercambio de los ángulos de los voltajes complejos nodales y

b) inclusión del efecto capacitivo de líneas transmisión en el modelo de red.

En este trabajo se presenta un análisis de la asignación de costos por uso de la red mediante la aplicación de factores lineales de participación y el método de MW-Milla. Dos métodos alternativos para reducir la dependencia del nodo compensador en la obtención de los factores de participación son presentados, los cuales están basados en ideas relativamente simples y aplicadas en la resolución de problemas de ingeniería eléctrica.

\section{Factores lineales de participación}

\section{Factores de participación convencionales}

Los FP, denotados como $a_{l j}$, son definidos como:

$$
a_{l j}=a_{i-m, j}=\frac{\Delta f_{l}}{\Delta P_{j}}=\frac{\Delta f_{i-m}}{\Delta P_{j}}
$$

donde $l$ representa el $l$-ésimo elemento de transmisión, el cual conecta a los nodos $i \mathrm{y} m$, jes el jésimo nodo del sistema.

En esta definición, se supone que $\Delta P_{j}$ es compensado exactamente por un total de $\Delta P_{j}$, (considerando $\Delta P_{L}=0$, es decir, cero cambios en las pérdidas), por el nodo compensador del sistema eléctrico. Entonces, 
$a_{\text {i }}$ representa la sensibilidad del flujo en la línea o transformador $l$, que conecta a los nodos $i$ y $m$, ante un cambio de potencia activa en el nodo $j$.

Supóngase que se desea estudiar la salida de un generador y que tal pérdida es absorbida por el nodo compensador (referencia). Si el nodo en cuestión está generando $P_{j}^{0} \mathrm{MW}$, su pérdida puede representarse como:

$$
\Delta P_{j}=-P_{j}^{0}
$$

y el nuevo flujo de potencia, $f_{l}^{\text {nuevo }}$, en cada elemento de transmisión del sistema podrá determinarse, a partir del flujo inicial, $f_{l}^{0}$, mediante la expresión:

$$
f_{l}^{\text {nиео o }}=f_{l}^{0}+a_{l j} \Delta P_{j} \quad 1=1, \ldots, n_{l}
$$

Si se tiene precalculados tales factores, estos se pueden aplicar para verificar los cambios, en forma aproximada, de flujo de potencia activa en todos los elementos de transmisión.

Los FP convencionales son calculados a partir del modelo lineal de flujos de potencia, conocido como flujos de C.D., el cual se representa matemá ticamente como:

$$
\left[\begin{array}{l}
P_{2} \\
\vdots \\
P_{n}
\end{array}\right]=\left[\begin{array}{lll} 
& & \\
\quad & &
\end{array}\right]\left[\begin{array}{l}
\theta_{2} \\
\vdots \\
\theta_{n}
\end{array}\right]
$$

donde se considera que el nodo 1 es la referencia y además,

$$
B_{i n}=-\frac{1}{x_{i m}}, i, m \neq 1 ; \quad B_{i i}=\sum_{m \in i} \frac{1}{x_{i m}}, \quad i \neq 1
$$

donde $m \in i$ indica todos los $m$ nodos conectados directamente a través de elementos de transmisión incidentes al nodo $i$.

Resolviendo para los ángulos de fase:

$$
[\theta]=[F][P]
$$

donde $[F]=[B]^{-1}$. Con esta matriz, se determinan los factores de participación, los cuales relacionan flujos de potencia activa en la red lineal, con las inyecciones de potencia activa, tanto de generadores como de cargas. Sea el factor de participación de la inyección de potencia en el nodo $j$, denotada como $P_{j}$ en el flujo del elemento $i-m$. Entonces, este puede calcularse de la manera siguiente:

$$
a_{l j}=\frac{1}{x_{l}}\left(\frac{d \theta_{i}}{d P_{j}} \frac{d \theta_{m}}{d P_{j}}\right)=\frac{1}{x_{l}}\left(F_{i j}-F_{m j}\right)
$$

donde $i$ y $m$ son nodos de envío y de recepción del elemento de transmisión $l, \mathrm{y}$, además, indican renglones de la matriz $[F]$; $x_{i n}$ es la reactancia de la línea de transmisión entre los nodos $i \mathrm{y} m$.

\section{Permutación de ángulos}

A fin de eliminar la dependencia del nodo compensador, se puede ejecutar un procedimiento de permuta de ángulos de los voltajes complejos nodales, de modo que los flujos de potencia correspondan exactamente a los obtenidos en el caso base de estudio, además de que el nodo con el ángulo de referencia, igual a cero grados, puede ser cualquiera que no tenga carga ni generación asociadas, esto es, cualquier nodo que represente a una subestación de paso en el sistema (Strbac et al., 1997) . 
Aplicación de los factores de participación y del método de MW-Milla en la asignación de ...

$$
\boldsymbol{\theta}_{i}^{P}=\boldsymbol{\theta}_{i}-\boldsymbol{\theta}_{r f}
$$

donde $\theta_{i}^{P}$ es el ángulo de voltaje nodal del $i$-ésimo nodo a permutarse y $\theta_{\text {ref }}$ es el ángulo de voltaje nodal del nodo de referencia.

\section{Inclusión del efecto capacitivo}

El método de FP incluyendo el efecto capacitivo consiste en recalcular la matriz de coeficientes [B] considerando el efecto capacitivo de las líneas de transmisión, lo cual implica que los elementos diagonales de esta matriz son modificados, de acuerdo a lo siguiente:

$$
B_{i i}^{\text {Nuevo }}=B_{i i}^{\text {Anterior }}+\sum_{m \in i} b_{m, 0}
$$

donde $b_{m, 0}$ es la semi-susceptancia del elemento de transmisión $i-m$. Esta modificación puede hacerse más general si se incluyen elementos de compensación en derivación y transformadores con cambiador de derivación fuera del nominal.

\section{Método de MW-Milla}

En esta sección se desarrollan las expresiones del método de MW-Milla en función de los FP. El método de MW-Milla asigna costos a un usuario $u$ de la red de la siguiente manera:

$$
R(u)=\sum_{l=1}^{n l} C_{l} \frac{f_{1}(u)}{f_{l}}
$$

donde $R(u)$ es el costo por uso de red del participante $u, C_{l}$ es el costo total anual (\$/año) del elemento $l, f_{l}(u)$ es el flujo en el elemento $l$ debido a la inyección de potencia de $u$ y $f_{l}$ es el flujo neto (actual o medido) del elemento $l$.
En función de los FP y de las inyecciones de potencia, el flujo a través del elemento $l$ puede expresarse como:

$$
f_{l}=\sum_{j=1}^{n} a_{l, j} P_{j}
$$

De la ecuación (11) resulta claro que la aportación del agente $u$ al flujo del elemento $l$ es:

$$
f_{l}(u)=a_{l, u} P_{u}
$$

Entonces, el cargo al agente $u$ por usar el elemento $l$, aplicando el método de MW-Milla es:

$$
R(u)=\sum_{l=1}^{n l} C_{l} \frac{a_{l, u} P_{u}}{\sum_{j=1}^{n} a_{l, j} P_{j}}=\sum_{l=1}^{n l} C_{l} \frac{a_{l, u} P_{u}}{f_{l}}
$$

donde $n_{l}$ es el número de elementos de la red de transmisión

En la literatura se emplean tres criterios basados en este método para evaluar el costo por uso de la red de transmisión, los cuales son:

Criterio A. Al total de costos por incrementos, restar los costos por decrementos de flujos. La ecuación (13) es la que se aplica para este criterio.

Criterio B. Cada costo individual por cambio de flujo, convertirlo a valor absoluto y, posteriormente, determinar la suma total de los mismos.

$$
R(u)=\sum_{l=1}^{n l} C_{l} \frac{a_{l, u} P_{u}}{\sum_{j=1}^{n} a_{l, j} P_{j}}
$$


Criterio $C$. Únicamente se considera los costos relacionados con incrementos de flujo:

$$
R(u)=\sum_{l=1}^{n l} C_{l} \frac{a_{l, u} P_{u}}{\sum_{j=1}^{n} a_{l, j} P_{j}}
$$

donde:

$a_{l, j}=\left\{\begin{array}{cc}a_{l, j} & \text { Si el factor es el mismo signo que el flujo } \\ 0 & \text { Si el factor es el mismo que el flujo }\end{array}\right.$

\section{Caso de estudio}

En esta sección se presenta un ejemplo de aplicación para la asignación de cargos por usos de redes de transmisión. Para esto, se utiliza el sistema de seis nodos de Word y Wollenberg (1996). El costo de cada elemento de transmisión se considera unitario (1.0 Miles de \$/mes).

\section{A. FP convencionales}

La tabla 1 presenta los FP considerando al nodo 1 como referencia.
Debido a que los FP del nodo compensador son cero, esto implica que los cargos por uso de la red de transmisión sean nulos. Ante esta situación, el generador no pagaría por uso de la red de transmisión, distribuyéndose los costos de la red entre los demá s participantes del sistema. Los cargos por uso de la red elemento a elemento, a través de los criterios $\mathrm{B}$ y C del método de MW-Milla, se muestran en la Tabla 2.

De la tabla 2, se observa que solo hay cargo por uso de red para los nodos en donde hay generació $\mathrm{n}$ o demanda. Se observa además que, en el caso del criterio $C$, hay valores cero en la asignación de costos elemento a elemento. Lo anterior, es resultado de que el criterio $\mathrm{C}$ no considera que el contraflujo a través de algún elemento de la red (valores de FP negativos) no causa asignación de cargos. Es en el nodo 4 donde esta condición se muestra con mayor efecto en la asignación de costos. En el criterio B, los costos asociados al nodo 4 son de 2.6131 Miles $\$$ /mes mientras que la aplicación del criterio $C$ da un valor de 4.0826, lo que representa alrededor de un incremento del $60 \%$. Por otro lado, el nodo 2 experimenta una disminución semejante cuando se aplica el criterio B y, posteriormente, el criterio C, mientras que el nodo 5 no presenta cambios significativos.

Tabla 1. FP del sistema de prueba (método convencional)

\begin{tabular}{ccccccc}
\hline Línea & Nodo 1 & Nodo 2 & Nodo 3 & Nodo 4 & Nodo 5 & Nodo 6 \\
\hline $1-2$ & 0.0 & -0.47062 & -0.40256 & -0.31488 & -0.32172 & -0.40642 \\
$1-4$ & 0.0 & -0.31448 & -0.29487 & -0.50437 & -0.27109 & -0.29600 \\
$1-5$ & 0.0 & -0.21448 & -0.30256 & -0.18073 & -0.40717 & -0.29756 \\
$2-3$ & 0.0 & 0.05444 & -0.34155 & 0.01601 & -0.10569 & -0.19066 \\
$2-4$ & 0.0 & 0.09926 & -0.03419 & 0.02919 & -0.19268 & -0.02551 \\
$2-5$ & 0.0 & 0.31146 & 0.21538 & -0.37897 & 0.10126 & 0.22083 \\
$2-6$ & 0.0 & 0.06419 & -0.24220 & 0.01888 & -0.12461 & -0.40998 \\
$3-5$ & 0.0 & 0.06217 & 0.28896 & 0.01828 & -0.12070 & 0.15263 \\
$3-6$ & 0.0 & -0.00773 & 0.36947 & -0.00227 & 0.01500 & -0.34300 \\
$4-5$ & 0.0 & -0.00342 & -0.07948 & 0.11664 & -0.16983 & -0.07516 \\
$5-6$ & 0.0 & -0.05646 & -0.12722 & -0.01660 & 0.10960 & -0.24671 \\
\hline
\end{tabular}


DOI: http://dx.doi.org/10.22201/fi.25940732e.2007.08n2.009

Aplicación de los factores de participación y del método de MW-Milla en la asignación de ...

Tabla 2. Cargos por uso de red (MW-MILLA criterio b y c)

\begin{tabular}{ccccccc}
\hline & \multicolumn{3}{c}{ Criterio B } & \multicolumn{3}{c}{ Criterio C } \\
\hline Línea & Nodo 2 & Nodo 4 & Nodo 5 & Nodo 2 & Nodo 4 & Nodo 5 \\
\hline $1-2$ & 0.3456 & 0.3237 & 0.3307 & 0 & 0.4946 & 0.5054 \\
$1-4$ & 0.2248 & 0.5042 & 0.2710 & 0 & 0.6504 & 0.3496 \\
$1-5$ & 0.2067 & 0.2439 & 0.5494 & 0 & 0.3074 & 0.6926 \\
$2-3$ & 0.2422 & 0.0997 & 0.6581 & 0.2690 & 0 & 0.7310 \\
$2-4$ & 0.3166 & 0.5393 & 0.1441 & 0.3699 & 0.6301 & 0 \\
$2-5$ & 0.2422 & 0.0997 & 0.6581 & 0.2690 & 0 & 0.7310 \\
$2-6$ & 0.2422 & 0.0997 & 0.6581 & 0.2690 & 0 & 0.7310 \\
$3-5$ & 0.2422 & 0.0997 & 0.6581 & 0.2690 & 0 & 0.7310 \\
$3-6$ & 0.2422 & 0.0997 & 0.6581 & 0 & 1.0000 & 0 \\
$4-5$ & 0.0085 & 0.4037 & 0.5878 & 0 & 0 & 1.0000 \\
$5-6$ & 0.2422 & 0.0997 & 0.6581 & 0 & 1.0000 & 0 \\
Total & 2.5554 & 2.6131 & 5.8318 & 1.4458 & 4.0826 & 4.4716 \\
\hline
\end{tabular}

La suma de los totales en cada criterio da el costo total de la red. Por ejemplo, para el criterio B (11 Miles \$/mes):

\section{$\mathrm{C}=2.5554+2.6131+5.8318=11.0$ Miles $\$$ /mes}

\section{B. Permutación de ángulos}

De la figura 1 , se observa que en los nodos 3 y 6 no existe generación ni carga asociadas.
Entonces, la permutación de ángulos puede realizarse suponiendo que $\theta_{3} \circ \theta_{6}$ son iguales a cero. Para estos casos, la tabla 3 muestra los resultados de la permutación, donde se nota que las diferencias angulares entre nodos adyacentes, con respecto al caso base, permanecen invariantes y que, por lo tanto, los flujos de potencia serán exactamente los mismos en ambos casos.

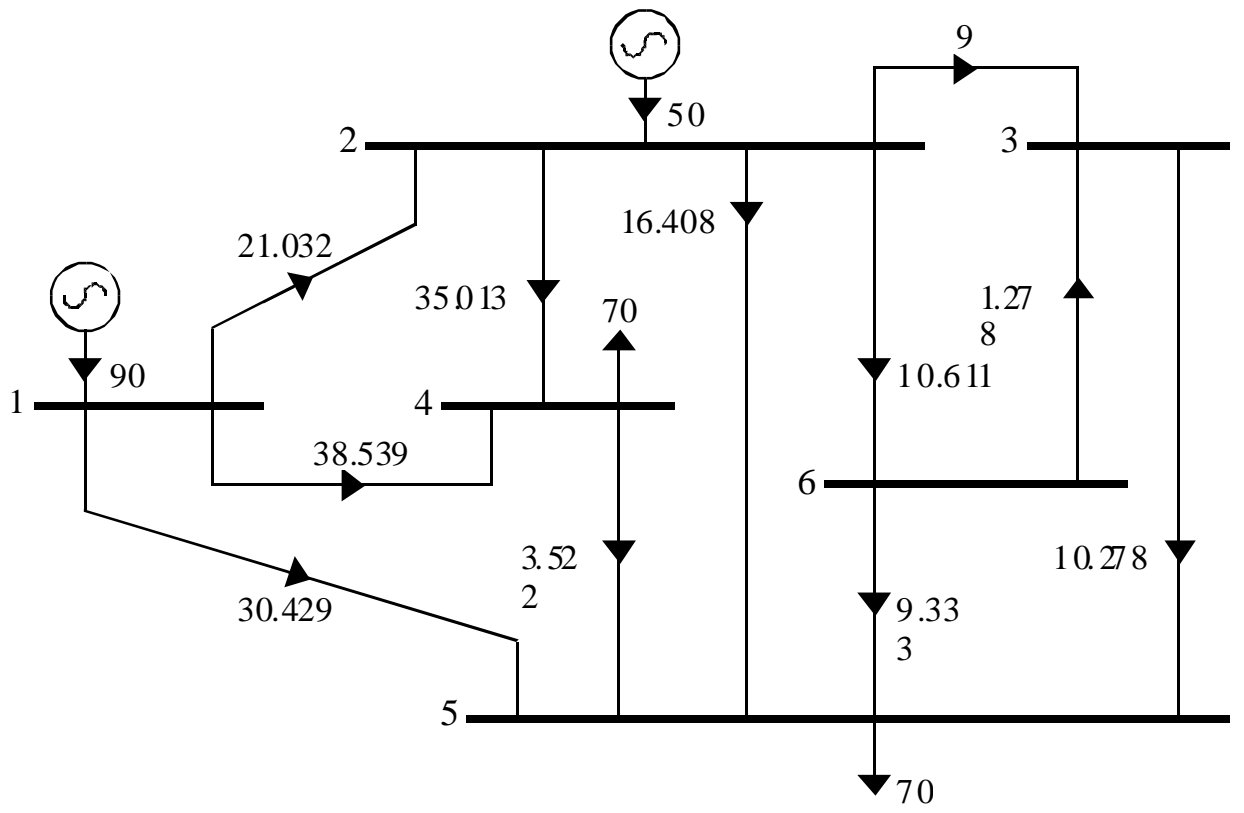

Figura 1. Sistema de prueba de seis nodos 
DOI: http://dx.doi.org/10.22201/fi.25940732e.2007.08n2.009

L. Alba-Gómez, J.H. Tovar-Hernández y G. Gutiérrez-Alcaraz

Tabla 3. FP del sistema de prueba (método convencional)

\begin{tabular}{cccc}
\hline Nodo & $\theta_{1}=0^{\circ}$ & $\theta_{3}=0^{\circ}$ & $\theta_{6}=0^{\circ}$ \\
\hline 1 & 0.000 & 3.699 & 3.626 \\
2 & -2.410 & 1.289 & 1.216 \\
3 & -3.699 & 0.000 & -0.073 \\
4 & -4.416 & -0.717 & -0.790 \\
5 & -5.230 & -1.531 & -1.604 \\
6 & -3.626 & 0.073 & 0.000 \\
\hline
\end{tabular}

La tabla 3 presenta los FP considerando que $\theta_{3}$ es el ángulo de referencia.

Tabla 3. FP del sistema de prueba (permutacion de angulos entre los nodos 1 y 3)

\begin{tabular}{cccccccc}
\hline Línea & Nodo 1 & Nodo 2 & Nodo 3 & Nodo 4 & Nodo 5 & Nodo 6 \\
\hline $1-2$ & 0.40256 & -0.06806 & 0.0 & 0.08767 & 0.08083 & -0.00386 \\
$1-4$ & 0.29487 & -0.02001 & 0.0 & -0.20950 & 0.02377 & -0.00113 \\
$1-5$ & 0.30256 & 0.08807 & 0.0 & 0.12183 & -0.10460 & 0.00500 \\
$2-3$ & 0.34155 & 0.39600 & 0.0 & 0.35756 & 0.23585 & 0.15088 \\
$2-4$ & 0.03419 & 0.13345 & 0.0 & 0.06338 & -0.15849 & 0.00757 \\
$2-5$ & -0.21538 & 0.09608 & 0.0 & -0.59436 & -0.11411 & 0.00545 \\
$2-6$ & 0.24220 & 0.30639 & 0.0 & 0.26108 & 0.11758 & -0.16778 \\
$3-5$ & -0.28896 & -0.22678 & 0.0 & -0.27067 & -0.40966 & -0.13633 \\
$3-6$ & -0.36947 & -0.37721 & 0.0 & -0.37175 & -0.35447 & -0.71277 \\
$4-5$ & 0.07948 & 0.07606 & 0.0 & 0.19612 & -0.09034 & 0.00432 \\
$5-6$ & 0.12727 & 0.07081 & 0.0 & 0.11067 & 0.23688 & -0.11943 \\
\hline
\end{tabular}

Similar al caso anterior, para el nodo compensador los FP convencionales son cero. Sin embargo, la asignación de costos por uso de red para todas las inyecciones/extracciones es posible, lo cual se muestra en la tabla 4.

Tabla 4. Cargos por uso de red (MW-MILLA criterios B y C) (continúa...)

\begin{tabular}{cccccccccc}
\hline \multicolumn{4}{c}{ Criterio B } \\
\hline Línea & Nodo 1 & Nodo 2 & Nodo 4 & Nodo 5 & Nodo 1 & Nodo 2 & Nodo 4 & Nodo 5 \\
$1-2$ & 0.7045 & 0.0662 & 0.1193 & 0.1100 & 1.0000 & 0 & 0 & 0 \\
$1-4$ & 0.6049 & 0.0228 & 0.3343 & 0.0379 & 0.6441 & 0 & 0.3559 & 0 \\
$1-5$ & 0.5735 & 0.0927 & 0.1796 & 0.1542 & 0.6990 & 0.1130 & 0 & 0.1880 \\
$2-3$ & 0.3338 & 0.2150 & 0.2718 & 0.1793 & 0.6082 & 0.3918 & 0 & 0 \\
\hline
\end{tabular}


DOI: http://dx.doi.org/10.22201/fi.25940732e.2007.08n2.009

Aplicación de los factores de participación y del método de MW-Milla en la asignación de ...

Tabla 4. Cargos por uso de red (MW-MILLA criterios B y C) (...continuación)

\begin{tabular}{cccccccccc}
\hline & \multicolumn{4}{c}{ Criterio B } & \multicolumn{5}{c}{ Criterio C } \\
\hline Línea & Nodo 1 & Nodo 2 & Nodo 4 & Nodo 5 & Nodo 1 & Nodo 2 & Nodo 4 & Nodo 5 \\
\hline $2-4$ & 0.2627 & 0.0651 & 0.5639 & 0.1083 & 0 & 0.0883 & 0.7648 & 0.1468 \\
$2-5$ & 0.1217 & 0.2639 & 0.1755 & 0.4388 & 0.1476 & 0.3201 & 0 & 0.5323 \\
$2-6$ & 0.3426 & 0.2408 & 0.2872 & 0.1294 & 0.5873 & 0.4127 & 0 & 0 \\
$3-5$ & 0.3061 & 0.1335 & 0.2230 & 0.3375 & 0 & 0 & 0.3979 & 0.6021 \\
$3-6$ & 0.3230 & 0.1832 & 0.2528 & 0.2410 & 0 & 0 & 0.5119 & 0.4881 \\
$4-5$ & 0.2307 & 0.1226 & 0.4427 & 0.2039 & 0.4140 & 0.2201 & 0 & 0.3659 \\
$5-6$ & 0.2913 & 0.0900 & 0.1970 & 0.4217 & 0.7639 & 0.2361 & 0 & 0 \\
Total & 4.0948 & 1.4959 & 3.0472 & 2.3621 & 4.0644 & 1.4716 & 3.0255 & 2.4385 \\
\hline
\end{tabular}

La tabla 5 muestra los FP convencionales cuando se hace la permutación angular entre los nodos 1 y 6 , mientras que la tabla 6 presenta las asignaciones de cargos para las inyecciones/extracciones de potencia del sistema.

Tabla 5. FP del sistema de prueba (permutacion de angulos entre los nodos 1 y 6)

\begin{tabular}{ccccccc}
\hline Línea & Nodo 1 & Nodo 2 & Nodo 3 & Nodo 4 & Nodo 5 & Nodo 6 \\
\hline $1-2$ & 0.40642 & -0.6419 & 0.00386 & 0.09153 & 0.08469 & 0.0 \\
$1-4$ & 0.29600 & -0.01888 & 0.00113 & -0.20837 & 0.02491 & 0.0 \\
$1-5$ & 0.29756 & 0.083307 & -0.00500 & 0.11683 & -0.10960 & 0.0 \\
$2-3$ & 0.19066 & 0.24511 & -0.15088 & 0.20668 & 0.08497 & 0.0 \\
$2-4$ & 0.02661 & 0.12587 & -0.00757 & 0.05580 & -0.16607 & 0.0 \\
$2-5$ & -0.22083 & 0.09062 & -0.00545 & -0.59981 & -0.11957 & 0.0 \\
$2-6$ & 0.40998 & 0.47418 & 0.16778 & 0.42886 & 0.28537 & 0.0 \\
$3-5$ & -0.15263 & -0.09045 & 0.13633 & -0.13434 & -0.27333 & 0.0 \\
$3-6$ & 0.34330 & 0.33556 & 0.71277 & 0.34102 & 0.35830 & 0.0 \\
$4-5$ & 0.07516 & 0.07174 & -0.00432 & 0.19180 & -0.090034 & 0.0 \\
$5-6$ & 0.24671 & 0.19024 & 0.11943 & 0.23010 & 0.35632 & 0.0 \\
\hline
\end{tabular}

Tabla 6. Cargos por uso de red (MW-MILLA criterio B y C) (continúa...)

\begin{tabular}{ccccccccc}
\hline \multicolumn{1}{c}{ Criterio B } \\
\hline Línea & Nodo 1 & Nodo 2 & Nodo 4 & Nodo 5 & Nodo 1 & Nodo 2 & Nodo 4 & Nodo 5 \\
\hline $1-2$ & 0.7017 & 0.0616 & 0.1229 & 0.1137 & 1.0000 & 0 & 0 & 0 \\
$1-4$ & 0.6066 & 0.0215 & 0.3321 & 0.0397 & 0.6462 & 0 & 0.3538 & 0 \\
$1-5$ & 0.5724 & 0.0888 & 0.1748 & 0.1640 & 0.6937 & 0.1076 & 0 & 0.1987 \\
$2-3$ & 0.3444 & 0.2459 & 0.2903 & 0.1194 & 0.5834 & 0.4166 & 0 & 0 \\
$2-4$ & 0.2658 & 0.0606 & 0.5616 & 0.1120 & 0 & 0.0826 & 0.7650 & 0.1525 \\
$2-5$ & 0.0989 & 0.2599 & 0.1613 & 0.4800 & 0.1179 & 0.3098 & 0 & 0.5723 \\
\hline
\end{tabular}


DOI: http://dx.doi.org/10.22201/fi.25940732e.2007.08n2.009

L. Alba-Gómez, J.H. Tovar-Hernández y G. Gutiérrez-Alcaraz

Tabla 6. Cargos por uso de red (MW-MILLA criterio B y C) (...continuación)

\begin{tabular}{ccccccccc}
\hline \multicolumn{9}{c}{ Criterio B } \\
\hline Línea & Nodo 1 & Nodo 2 & Nodo 4 & Nodo 5 & Nodo 1 & Nodo 2 & Nodo 4 & Nodo 5 \\
\hline $2-6$ & 0.3336 & 0.2144 & 0.2714 & 0.1806 & 0.6088 & 0.3912 & 0 & 0 \\
$3-5$ & 0.2935 & 0.0966 & 0.2010 & 0.4089 & 0 & 0 & 0.3295 & 0.6705 \\
$3-6$ & 0.3197 & 0.1736 & 0.2470 & 0.2596 & 0.6481 & 0.3519 & 0 & 0 \\
$4-5$ & 0.2225 & 0.1180 & 0.4416 & 0.2179 & 0.3984 & 0.2113 & 0 & 0.3903 \\
$5-6$ & 0.3051 & 0.1307 & 0.2214 & 0.3428 & 0.7001 & 0.2999 & 0 & 0 \\
Total & 4.0642 & 1.4716 & 3.0254 & 2.4386 & 5.3966 & 2.1709 & 1.4483 & 1.9843 \\
\hline
\end{tabular}

En ambos casos de permutación, ahora se tiene una redistribución de cargos, debido a que ahora el nodo compensador tiene cargos diferentes de cero. Sin embargo, note que los resultados para los dos casos de permutación son distintos entre sí, los cuales dependen de la localización del nodo de referencia (Tabla 7). Entonces, aquí el punto bajo discusión sería una localización neutral del nodo de referencia, pudiéndose no llegar a acuerdo alguno.
C. Inclusión del efecto capacitivo

Los FP son presentados en la tabla 8. En este caso, debido a que los FP del nodo de referencia son distintos de cero, habrá cargos por uso de red para la generación localizada de ese nodo.

Los cargos por uso de la red de transmisión, para los mé todos MW-Milla, Criterios B y C son presentados en la tabla 9.

Tabla 7. Cargos por uso de red (MW-MILLA criterio B y C)

\begin{tabular}{ccccc}
\hline & \multicolumn{3}{c}{$\theta_{3}=0^{\circ}$} & $\theta_{6}=0^{\circ}$ \\
\hline Criterio B & Criterio C & Criterio B & Criterio C \\
\hline Nodo 1 & 4.0948 & 4.0644 & 4.8640 & 5.3965 \\
Nodo 2 & 1.4959 & 1.4716 & 1.7822 & 2.1709 \\
Nodo 4 & 3.0472 & 3.0255 & 2.0305 & 1.4483 \\
Nodo 5 & 2.3621 & 2.4385 & 2.3233 & 1.9842 \\
Total & 11 & 11 & 11 & 11 \\
\hline
\end{tabular}

Tabla 8. FP del sistema de prueba (inclu sion del efecto capacitivo) (continúa...)

\begin{tabular}{ccccccc}
\hline Línea & Nodo 1 & Nodo 2 & Nodo 3 & Nodo 4 & Nodo 5 & Nodo 6 \\
\hline $1-2$ & 0.3266 & -0.1420 & -0.0735 & 0.0130 & 0.0065 & -0.0774 \\
$1-4$ & 0.2798 & -0.0337 & -0.0137 & -0.2228 & 0.0097 & -0.0149 \\
$1-5$ & 0.2546 & 0.0412 & -0.0458 & 0.0746 & -0.1500 & -0.0409 \\
$2-3$ & 0.0819 & 0.1367 & -0.2573 & 0.0979 & -0.0226 & -0.1068 \\
$2-4$ & -0.0936 & 0.2165 & 0.1195 & -0.4716 & 0.0065 & 0.1250 \\
$2-5$ & 0.0369 & 0.1359 & 0.0032 & 0.0659 & -0.1543 & 0.0107 \\
$2-6$ & 0.0996 & 0.1642 & -0.1401 & 0.1185 & -0.0235 & -0.3075 \\
\hline
\end{tabular}


DOI: http://dx.doi.org/10.22201/fi.25940732e.2007.08n2.009

Aplicación de los factores de participación y del método de MW-Milla en la asignación de ...

Tabla 8. FP del sistema de prueba (inclu sion del efecto capacitivo) (...continuación)

\begin{tabular}{ccccccc}
\hline Línea & Nodo 1 & Nodo 2 & Nodo 3 & Nodo 4 & Nodo 5 & Nodo 6 \\
\hline $3-5$ & -0.0361 & 0.0254 & 0.2511 & -0.0181 & -0.1563 & 0.1150 \\
$3-6$ & -0.0055 & -0.0132 & 0.3630 & -0.0078 & 0.0093 & -0.3481 \\
$4-5$ & 0.0511 & 0.0478 & -0.0275 & 0.1673 & -0.1173 & -0.0232 \\
$5-6$ & 0.0295 & -0.0264 & -0.0966 & 0.0131 & 0.1386 & -0.2157 \\
\hline
\end{tabular}

Tabla 9. Cargos por uso de red (MW-MILLA criterio B y C)

\begin{tabular}{ccccccccc}
\hline \multicolumn{9}{c}{ Criterio B } \\
\hline Línea & Nodo 1 & Nodo 2 & Nodo 4 & Nodo 5 & Nodo 1 & Nodo 2 & Nodo 4 & Nodo 5 \\
\hline $1-2$ & 0.7763 & 0.1875 & 0.0241 & 0.0120 & 1.0000 & 0 & 0 & 0 \\
$1-4$ & 0.5836 & 0.0391 & 0.3615 & 0.0158 & 0.6175 & 0 & 0.3825 & 0 \\
$1-5$ & 0.5631 & 0.0507 & 0.1283 & 0.2579 & 0.6460 & 0.0581 & 0 & 0.2959 \\
$2-3$ & 0.3256 & 0.3019 & 0.3027 & 0.0698 & 0.4669 & 0.4330 & 0 & 0.1001 \\
$2-4$ & 0.1597 & 0.2054 & 0.6263 & 0.0086 & 0 & 0.2469 & 0.7531 & 0 \\
$2-5$ & 0.1301 & 0.2662 & 0.1807 & 0.4230 & 0.1588 & 0.3249 & 0 & 0.5163 \\
$2-6$ & 0.3306 & 0.3028 & 0.3058 & 0.0608 & 0.4762 & 0.4362 & 0 & 0.0876 \\
$3-5$ & 0.1944 & 0.0759 & 0.0756 & 0.6540 & 0 & 0.0942 & 0.0939 & 0.8119 \\
$3-6$ & 0.2107 & 0.2809 & 0.2310 & 0.2774 & 0 & 0 & 1.000 & 0 \\
$4-5$ & 0.1708 & 0.0888 & 0.4352 & 0.3052 & 0.3024 & 0.1572 & 0 & 0.5404 \\
$5-6$ & 0.1819 & 0.0905 & 0.0627 & 0.6649 & 1.0000 & 0 & 0 & 0 \\
Total & 3.6268 & 1.8897 & 2.7339 & 2.7494 & 4.6678 & 1.7505 & 2.2295 & 2.3522 \\
\hline
\end{tabular}

De la tabla anterior se puede observar que el nodo 1 es el que presenta una mayor asignación de cargos para ambos criterios, lo cual es debido a que tiene la generación mayor en el sistema y a que FP relacionados con las lineas de transmisión 1-2, 1-4 y 1-5 tienen una magnitud relativamente mayor que las correspondientes a otros nodos (solo los nodos 3 y 6 tienen factores con magnitudes semejantes pero no tienen generación ni carga, por lo que no causan cargos). De aquí, se puede observar la importancia de aplicar un modelo de red adecuado para el cálculo de FP.

La tabla 10 presenta un resumen de los cálculos de cargos utilizando los criterios B y C del método MW-Milla y los diferentes métodos de cálculo de factores de participación. Puede notarse que en todos los casos hay resultados diferentes, aunque puede decirse que el método convencional no asigna cargos al nodo que introduce la mayor cantidad de potencia al sistema. La permutación angular elimina este problema, pero causa otro: decidir en dónde localizar al nodo de referencia. El método de factores de participación incluyendo el efecto capacitivo (FPIEC) asigna cargos a todos los nodos donde hay carga y generación, pero no existe la dependencia con respecto a la localización del nodo de referencia. 
Tabla 10. Cargos por uso de red mediante los diferentes métodos y modelos.

\begin{tabular}{ccccccccc}
\hline & \multicolumn{2}{c}{$\theta_{1}=\left.0\right|^{\circ}$} & \multicolumn{2}{c}{$\theta_{3}=\left.0\right|^{\circ}$} & \multicolumn{2}{c}{$\theta_{6}=\left.0\right|^{\circ}$} & \multicolumn{2}{c}{ FPIEC } \\
\hline & Criterio B & Criterio C & Criterio B & Criterio C & Criterio B & Criterio C & Criterio B & Criterio C \\
\hline Nodo 1 & 0 & 0 & 4.0948 & 4.0644 & 4.8640 & 5.3965 & 3.6268 & 4.6678 \\
Nodo 2 & 2.5554 & 1.4458 & 1.4959 & 1.4716 & 1.7822 & 2.1709 & 1.8897 & 1.7505 \\
Nodo 4 & 2.6131 & 4.0826 & 3.0472 & 3.0255 & 2.0305 & 1.4483 & 2.7339 & 2.2295 \\
Nodo 5 & 5.8318 & 5.4716 & 2.3621 & 2.4385 & 2.3233 & 1.9842 & 2.7494 & 2.3522 \\
Total & 11 & 11 & 11 & 11 & 11 & 11 & 11 & 11 \\
\hline
\end{tabular}

\section{Conclusiones}

En este trabajo se ha presentado el uso de los FP con el mé todo de MW-Milla, criterios B y C, para la asignación del cargo fijo por uso de redes de transmisión en mercados de electricidad. Como se ha hecho notar, los FP son dependientes de $\mathrm{b}$ localización del nodo compensador, lo cual ha sido motivo de controversia en la aplicación de metodologías para la asignación de cargos por uso de redes. Una solución parcial es la aplicación del método de permutació $n$ angular, aunque esto origina un problema de localización de un nodo sin carga y generación $\mathrm{y}$, en sistemas muy grandes, como es el caso del Sistema Eléctrico de México, esto puede ser también causa de controversia.

La inclusión del efecto capacitivo de las líneas de transmisión para calcular los elementos diagonales de la matriz [B] incluyendo el nodo de referencia, esto es con el fin de realizar el cargo a todos los participantes del sistema sin que se tenga una dependencia del nodo compensador se ha presentado y analizado.

\section{Agradecimientos}

El autor principal agradece al CONACyT el apoyo económico para la realización de sus estudios de maestría y al apoyo por parte de la DGEST para el desarrollo de este trabajo de investigación a través del proyecto 472.05-P.

\section{Referencias}

Alexander G. y Muñoz C. (2006). The New Chilean Transmission Charge Scheme as Compared with Current Allocation Methods, IEEE Trans. On Power Systems, Vol. 21, No. 1, pp 99-107.

Aswhani K. y Srivastava S., AC Power Transfer Distri bu tion Factors for Allocating Power Transactions in a Deregulated Market,

Bialek J. (1997), Topological Generation and Load Distribution Factors for Supplement Charge Allocation in Transmission Open Access, IEEE Trans. On Power Systems, Vol. 12, No. 3, pp1185-1193.

Happ H. (1994). Cost of Wheeling Methodologies, IEEE Trans. On Power Systems, Vol.9, No.1, pp. 147-156.

Joong S., Kern-Joong K. (2002). Reconstruction of the Jacobian Matrix by Angle Reference Transposition and Application to the New Penalty Factor Calculation, IEEE Power Engineering Review, pp 47-50.

Kankar B., Math H., y Jaap E. (2001).Operation of Restructured Power Systems, Kluwer Academic Publishers.

Kovacs R. y Leverett A. (1994). “A Load Flow Based Method for Calculating Embedded, Incre mental, and Marginal Cost of Transmission Capacity", IEEE Trans. On Power Systems, Vol. 9, No. 1. 
Marangon J. (1995). “Allocation of Transmis sion Fixed Charges: An Over view", IEEE/PES Summer Meeting, Portland, OR., pp 1409-1418.

Marangon J., Pereira M. y Pereira J. (1995). An Integrated Framework for Cost Allocation in a Multi-Owned Transmission System, IEEE Trans. On Power Systems, Vol.10, No.2, pp. 971-977.

Rudnick H., Palma R. y Fernández J. (1995). Marginal Pricing and Supplement Cost Allocation in Transmission Open Access, IEEE Trans. on Power Systems, Vol.10, No.2, pp 1125-1142

Shirmohammadi D., Vieira X., Gorestin B. y Pereira M. (1996). Some Fundamental Technical Concepts about Cost Based Transmission Pricing, IEEE Trans. On Power Systems, Vol.11, No.2, pp 1002-1008.
Shirmohammadi D., Gribik P., Law E., Malinowski J. y O'Donnell R. (1998), Evaluation of Transmission Network Capacity Use for Wheeling Transactions, IEEE Trans. On Power Systems, Vol.4, No.4, pp. 1405-1413.

Strbac G., Kirschen D. y Amhed S. (1997), Allocating Trans mis sion Usage on the Basis of Traceable Contributions of Generators and Loads to Flows, PE-222-PWRS-0-01, pp 527-534.

Vojdani C., Imparato N., Saini B., Wollenberg B. y Happ H. (1995). Trans mis sion Access Issues, IEEE/PES Winter Meeting, New York, N.Y., pp 41-51.

Wood A., Wollenberg B. (1996) Power Generation, Operation and Control, John Wiley \& Sons, Second Edition

\section{Semblanza de los autores}

Luís Alba-Gómez. Realizó sus estudios de licenciatura y maestría en el Instituto Tecnológico de Morelia (ITM), obteniendo los grados respectivos en 2004 y en 2006.

José Horacio Tovar-Hernández Realizó sus estudios de licenciatura en el Instituto Tecnológico de Morelia (ITM), obteniendo el grado de ingeniero electricista en 1984. Realizó sus estudios de maestría y doctorado en ingeniería eléctrica en la Sección de Estudios de Posgrado e Investigación de la Escuela Supe rior de Ingeniería Mecánica y Eléctrica del Instituto Politécnico Nacional, obteniendo los grados respectivos en 1989 y en 1995. Actualmente es profesor del programa de Graduados e Investigación en Ingeniería Eléctrica (PGIIE) del ITM. Guillermo Gutiérrez-Alcaraz. Realizó sus estudios de licenciatura y maestría en el Instituto Tecnológico de Morelia, obteniendo el grado de ingeniero y de maestro en ciencias, ambos en ingeniería eléctrica en 1995 y 1996 , respectivamente. Actualmente es profesor del PGIIE del ITM. 